\title{
Study on an Integrated Agent-based and Spatial Analysis Modelling for Energy- efficiency and Demand Analysis in Urban Planning
}

1 Yu SUN School of Architecture, Harbin Institute of Technology, Key Laboratory of Cold Region Urban and Rural Human Settlement Environment Science and Technology, Ministry of Industry and Information Technology, Harbin, China;

2 Hong LENG School of Architecture, Harbin Institute of Technology, Harbin 150000, China;

3 Tian WEI Mechanical Engineering, Tianjin University of Science and Technology, Tianjin , China;

\begin{abstract}
Cities and towns account for more than two-thirds of world energy consumption, a significant proportion of which is spent on operating buildings. Ambitious national energy and emission reduction targets necessitate that energy demand due to buildings is considered as an important measure when any future evolution of a district or city is planned. Energy consumption of buildings in cities is influenced by their immediate local environment. Factors such as local temperatures, wind speed (street-canyon effect), air pollution levels, human activities, access to daylight, etc. Indeed, in order to reduce energy consumption and associated carbon emissions globally, more attention should be focused on urban-scale energy analysis of the built environment. Nowadays, with the rise of the scientific paradigm shift and model theory, and the development of the spatial data, the use of the complex model of urban-system analysis become one of the important research of urban theory. Under this circumstance, this research will focus in the research stream, the application of a new integrated agent-based and spatial analysis modeling for energy prediction and energy-saving policy analysis in Urban Planning. The basis of the research will be to develop new, general purpose, computer models that can be used to assess the distribution of energy demand according to the spatial scale of the evaluated policy (e.g. local, city level). These models will look not only at the individual building level, but also at the district and city scales, in order to be able to assess the impact of urban planning policy and practice in land economy and spatial building pattern or design interventions on the energy demand of the wider scale.
\end{abstract}

\section{Keywords}

Spatial analysis, District energy efficiency, Urban planning.

\section{Introduction}

Cities and towns account for more than two-thirds of world energy consumption, a significant proportion of which is spent on operating buildings(Bose, 2013); (Robinson, 2011). Ambitious national energy and emission reduction targets necessitate that energy demand due to buildings is considered as an important measure when any future evolution of a district or city is planned. Not only the accounting of present-day energy consumption of 
the built environment by regions and by sectors, but also the prediction of achievable reductions to meet relevant emissions targets. (Keirstead, 2012). Indeed, in order to reduce energy consumption and associated carbon emissions globally, more attention should be focused on urban-scale energy analysis of the built environment (Heo and Choudhary, 2012).

Energy consumption of buildings in cities is influenced by their immediate local environment. Factors such as local temperatures, wind speed (street-canyon effect), air pollution levels, human activities, access to daylight, etc. Typically referred as micro-climatic factors, these are largely anthropogenic, resulting from local agglomerations of density of buildings and population, equipment, land-cover, activities of a city or region. In addition, many studies have proven that human behavior has a significant role in energy consumption patterns of buildings in urban settings (Frayssinet and Merlier, 2018). It is very likely behavior factors affecting energy use have positive spatial autocorrelation in city context. Therefore, it is important to consider spatial effects as well as occupants' behaviours in analysing energy consumption patterns of buildings in urban settings. However, there are still many limitations in current research technology and methodology, especially the interaction between energy consumption and occupant behavior in daily activities should be further discussed. Nowadays, with the rise of the scientific paradigm shift and model theory, and the development of the spatial data, the use of the complex model of urban-system analysis become one of the important researches of urban theory (Choudhary and Tian, 2014). Under this circumstance, the paper follows the technical waves, application of a new integrated agent-based and spatial analysis modeling for energy prediction and energy-saving policy analysis in Urban Planning. The basis of the research will be to develop new, general purpose, computer models that can be used to assess the distribution of energy demand according to the spatial scale of the evaluated policy (e.g. local, city level). The new integrated energy model that can be used to analyses energy consumption patterns due to buildings at the district scale, which also enables a technological innovation by ABM for behavior modeling.

We named the new integrated analysis environment: IUBEA from the initial letters of Integrated Urban Building Energy Analysis. The data used in the IUBEA includes physical features of buildings (e.g., size, volume) per use type and per district, demographics, economics (e.g., cost of land), and energy data at various spatial scales in cities. Another important feature of this computer model is that it is programmed within an open-source software (Netlogo), which allows other users to access it easily. In addition to being opensource, Netlogo (Tisue and Wilensky, 2004) is chosen in this project because it has many functions, such as scenario-based analysis, GIS environment, and agent-based modelling. Furthermore, it has several extensions to link with other programs, such as statistical program $R$ and numerical computing environment MATLAB.

In IUBEA, we implemented the model methods and validations through a case study of London. Agent-based modeling (ABM) in the integrated analysis environment (IUBEA) is a technique for bottom-up simulation, providing an alternative perspective to those that can be attained by using optimization or general-equilibrium approaches(Zhao and Wang, 2010) . This paper explores techniques to integrate a spatial analysis environment in the field of urban building energy assessment in cites to make full use of current spatial data relevant to urban-building energy consumption and energy efficiency policies. So, users of the model are able to easily relate building and spatial characteristics parameters to policy or utility 
figures, which can lead to an efficient energy model, which contributes the body of knowledge of energy modeling beyond the single building scale.

\section{The framework of the Integrated Agent-based and Spatial Analysis Modelling}

The main purpose of the research on urban scale building energy model is analysis the relationships and key factors affecting energy use in spatial autocorrelation in city context. Ensure that the planner can make reliable decision-making solutions based on system analysis of the mechanism of energy use and urban development. Techniques for extending building models to quantify energy consumption of a district, or city region have come into closer scrutiny as policy-implementers are under pressure to take large-scale actions for reducing energy consumed by regions, districts (Choudhary, 2012). In order to provide the reliable techniques for the low-carbon urban planning, researchers should use qualitative and quantitative analysis to evaluate the design of energy-saving policies before the implementation of energy-saving policy. On the one hand, to quantify the implementation effect of energy-saving strategies in different object's spatial position and scale. On the other hand, by comparing the environmental and socio-economic (include occupant behavior) impacts in spatial context, choose a more effective planning strategy that reduces the energy demand of district or city level. Therefore the typical steps for energy-efficiency analysis in urban planning as shown in Figure 1.

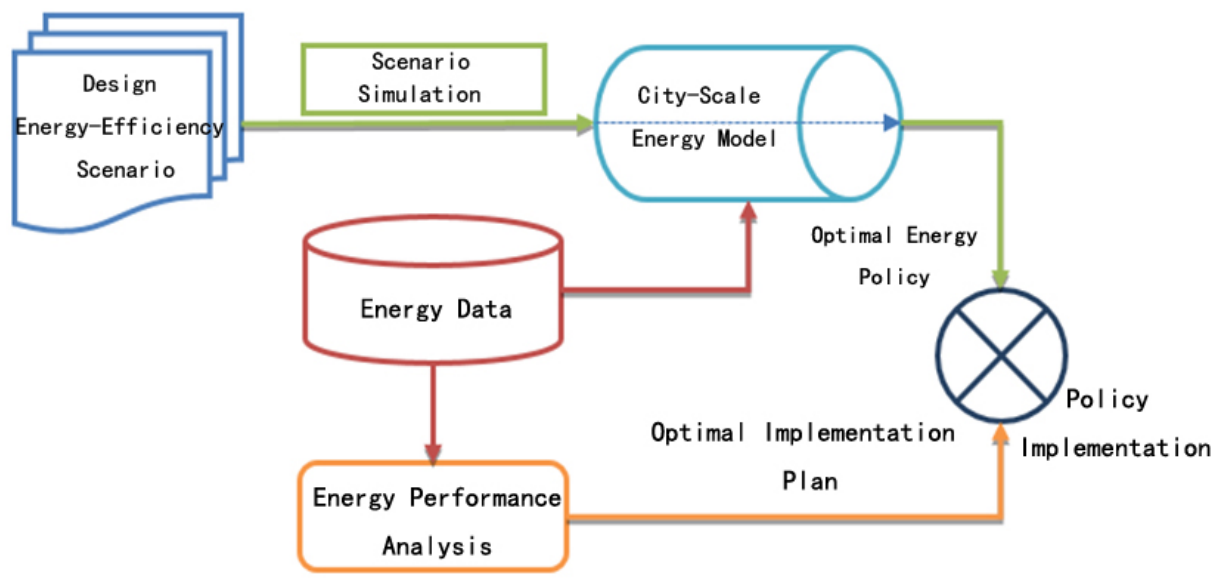

Figure 1 Composite relation of energy effectivity research

In addition, there are interactions and influence mechanisms of energy consumption and various spatial factors in built environment, named "spatial heterogeneity" (behaviour uncertainty caused by spatial position differences). By the influence the space phenomenon, policy-implementers should come into closer observation the energy-efficient in spatial to adopt different policies and implementation efforts in different regions to achieve the energy-saving purposes in the most economical way. Based on the above simulation key features, the systematic framework for the study of the integrated spatial analysis modelling for energy-efficiency and demand analysis in urban planning is constructed as shown in section3. 


\section{Contents and Methods in the Integrated Model Environment}

The analyses in IUEBA are implemented through four functions. The first displays the data spatially and can be used for data query at different spatial levels in cities. The second is to explore the relationship between various factors and energy consumption using correlation and regression analysis and create statistical energy models. The third is to study the effects of different scenarios on building energy use, named "scenario analysis". The fourth is to investigate the optimization zones using mixed-use method based on energy use intensity of districts. This section will describe these four main functions in detail.

\subsection{Spatial distribution analysis of the energy consumption in city scale in IUEBA}

These spreads (or distributions) can be used for estimating the probability distribution of the gross energy consumption per local authority in Greater London. The work is driven by the need to quantify future energy demand of buildings in their urban context as a function of projected growth of buildings and populations, refurbishments, policies incentivizing energy efficiency measures, and changes in building operation.

when predicting the energy consumption of a district in a city level, the actual value can vary significantly from the prediction, due to the different spatial contexts in which each district has its own unique characteristics that influence the energy consumption in various manners. The influence of the immediate environment and climate change on energy consumption or carbon emission in urban environment by the robust mathematical modeling method has been explored before (Cheng and Steemers, 2011). Geo-computation of existing distribution of energy consumption for an analysis of policy scenarios of energy use abatement is a case, where the context of the urban planning can be assessed and understood in detail, by gathering geospatial data, energy bill data and information using methods such as GIS and survey. Besides, advanced Geo-modeling methodologies of spatial analysis of energy consumption need be developed to improve the way in which observed data is used to spatial statistical test and validate models.

In this study the energy consumption of a set of uniform buildings within an area is normalized and expressed as $\mathrm{kWh} / \mathrm{m} 2 /$ year. The total energy consumption of buildings in a district can be expressed as the sum of energy consumption of its constituent buildings in the following form:

$$
E_{k}=\sum_{i=1}^{N} \operatorname{EUI}(i) \cdot P A(i, k)
$$

where, $E_{k}$ is the energy consumption of a district $\mathrm{k}$ in $\mathrm{kWh} / \mathrm{m} 2 /$ year, normalized by its total building floor area. EUI $(i$; is the energy use intensity of a building type $i$ in $\mathrm{kWh} / \mathrm{m} 2 /$ year, $P A(i, k)$ is the percentage of built floor area of building type $i$ in district $k$, and $N$ is the total number of building types.

A common feature in EUI studies is that even in the same type building, the difference of EUI is existence due to the varies in building age, sub-functions, and equipment performance. The hypothetical model does not fully reflect the actual energy consumption. So the energy density of 
different types of buildings is more appropriately expressed in the form of probability distribution show as (2).

$$
G_{k} \sim N\left(E_{k}, \sigma^{2}\right), \text { for } \mathrm{k}=1,2,3, \ldots, \mathrm{D}
$$

where, $G_{k}$ is the observed energy consumption of a district $\mathrm{k}$ in $\mathrm{kWh} / \mathrm{m}^{2} /$ year, $\mathrm{D}$ is the number of all observation points. $\sigma^{2}$ is the variance of the distribution, which represents the error in the measurement process, accommodating any differences between the recorded gross energy consumption of a district and that of its individual buildings. These errors may be systematic, owing to how energy consumption is recorded or due to other reasons in the observations.

There is also an external spatial influence value of building energy consumption in each district. Spatial analysis techniques of SAR and GWR is used to quantify spatial influence in energy consumption of a neighbourhood, district level.

We first use spatial autoregressive methods to incorporate spatial influences into the calculation of building energy density. The spatial feature quantity $b(k)$ is used to characterize the spatial characteristics of energy consumption in different regions as show in (3)

$$
E_{k}=\sum_{j=1}^{N} I_{j} P_{k, j}+b(k)+\alpha, \text { for } \mathrm{k}=1,2,3, \cdots, \mathrm{D}
$$

Where $\alpha$ is a constant term; however, the spatially distributed intercept $\alpha$ is varies in different regions, it is assumed that it satisfies a stable uniform distribution; $b(k)$ is a spatial feature of a certain district; It is expressed as the follow:

$$
b_{k} \mid b_{k-1} \sim N\left(\frac{1}{n_{k}} \sum_{j=1}^{n_{k}} b_{j}, \tau^{2}\right), \text { for } \mathrm{k}=1,2,3, \cdots, \mathrm{D}
$$

Where $n_{k}$ is the number of adjacent areas affected by the area $k ; \tau$ represents the accuracy of the distribution and is expressed in the model as follows:

$$
\tau=\frac{1}{\varphi^{2}}, \varphi \sim \text { uniform }(0,100) \mathrm{D}
$$

Where $\phi$ is the variance parameter obeys the uniform distribution : Uniform $(0,100)$.

A common feature in these studies is that the energy consumption of a set of uniform buildings within an area is normalized and expressed as $\mathrm{kWh} / \mathrm{m} 2 /$ year. This value, referred as energy use intensity (EUI), is derived from published standards of typical building consumption patterns, or from auditing energy consumption of a representative sample of buildings, or computed using statistical or physics-based energy models of representative buildings. It is a standard normalization because it enables scaling-up energy consumption of a large set of buildings into gross energy use of a district, and likewise, allows delegating total energy use of a district to its consumers (buildings).

\subsection{Spatial factors analysis of the energy consumption in city scale in IUEBA}

The immediate local environment has important influence on energy consumption of buildings which has been explored before. Such as local temperatures, wind, access to daylight, pollution, noise levels, etc. These spatial factors result in significant differences in the amount of energy use such heating, cooling, ventilation and lighting required to maintain a building at acceptable conditions. How to identify spatial influence factors, improve the accuracy of key factors, and eliminate the interference of non-critical factors on model predictions, is still a problem to be solved in the integrated environment. In this paper we use spatial statistical methods to quantify the influencing factors in the energy simulation. 
The regression analysis in this project has two main aims: interpretation and prediction. The interoperation is to understand the spatial relationship between energy and independent variables. For instance, how population number or floor area can influence the energy consumption and whether the floor area or population has more significant effects on energy consumption. The prediction is to investigate the effect of changed independent variables on energy consumption. Note that this regression analysis can deal with one or multiple independent variables. The regression analysis can be used for interpretation to understand the relative importance of independent variables on energy consumption. This analysis can also be very helpful to determine the variations of energy consumption explained by the independent variables in the regression.

The spatial form factors is shown as example how the regression analysis is implemented in IUBEA environment, as shown :

$$
\hat{E}_{t}=\text { Constant }+\beta_{\text {building }} \times S_{t}^{\text {building }}+\beta_{\text {road }} \times S_{t}^{\text {road }}+\cdots+\varepsilon
$$

where: $\hat{E}_{t}$ is estimated energy consumption of a given area, $\beta_{\text {building }}, \beta_{\text {road }}$, etc. are the coefficients of building, road, etc. $S_{t}^{\text {building }}, S_{t}^{\text {road }}$, etc. are the area of building, road, etc. $\varepsilon$ is the random error term.

Implement of correlation analysis on spatial socio-economic features with energy consumption. Normative building energy model has many parameters that may not be sensitive to analysis the Socio-economic features impact EUI distribution in spatial. The procedure of the Correlation and regression analysis is to apply a sensitivity analysis to a set of parameters to reveal the relations of distribution of Socio-source features with energy consumption in spatial.

The influence of the local environment on energy consumption of buildings has been explored Anthropogenic factor, such as local agglomerations of buildings and population types, equipment, activities, across various parts of a city as well as socio-economic features such as income levels, wealth, and demographics have also been shown to influence the energy consumption patterns in buildings. Given the feasible ranges of model variables, the next step is to generate data samples and retrieve the corresponding model outcomes for variable sensitivity analysis with the distribution of Socio-economic features. The method for sensitivity analysis is the same in different types of application in building energy analysis as show in Figure. 2 ( Tian, 2013).

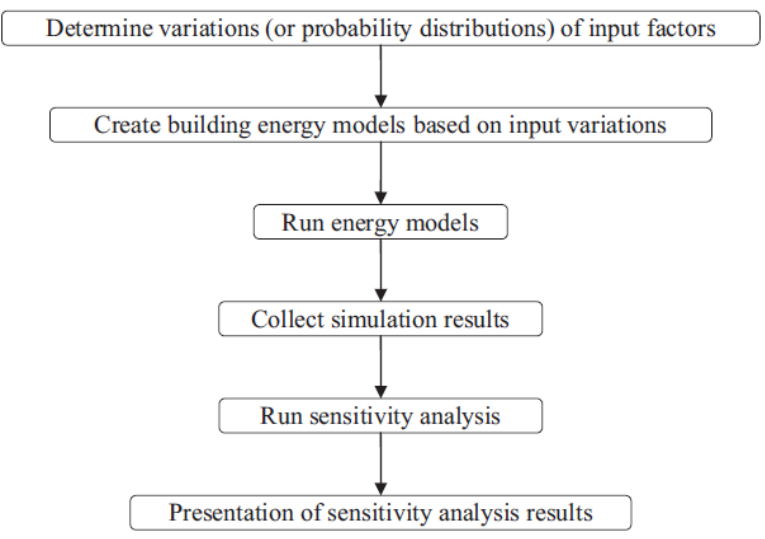

Figure 2 Flow diagram for sensitivity analysis in building performance analysis 


\subsection{Energy prediction and scenario analysis in city scale in IUEBA}

\section{Energy prediction}

Based on the analysis in section 3.1 and 3.2, the prediction analysis can be implemented if the independent variables can be used to explain most of variations of energy consumption. Then the regression model is used to predict the change of energy consumption due to the variations of independent variables. The total energy consumption of buildings in a district can be expressed as the sum of energy consumption of its constituent buildings in the following form

$$
E_{k}=\sum_{i=1}^{N} E U I(i) \cdot P A(i, k)
$$

The results from formula above should be verified with actual energy data to make sure that both energy use intensity and area data are reasonable at a given spatial scale. Then analysts can change energy use intensity or floor area to explore the possible effects of these changes on energy consumption in the same or different spatial scales in cities.

\section{Scenario analysis}

Baseline Scenario:

Scenario analysis is used for what-if analysis. The function in this section is different from "regression analysis", as explained in Section 3.3. Scenario analysis does not implement regression method, but it depends on only independent variables. Therefore, this method is more flexible compared to regression analysis because it is suitable for the situation where energy consumption data are unavailable in some spatial scales or some areas of a city. To implement the scenario analysis, analysts should have good knowledge on the effects of these independent variables on energy consumption. Furthermore, some form of validation of energy calculations is necessary to confirm that the prior assumptions in the model are appropriate.

New Scenario design:

This section will describe three new scenarios to explore the possible change of energy consumption in cities.

(1) Electrification Scenario: It seems there are more benefits for electrification in building sector if grid electricity can become gradually carbon intensive. As an example, electricity emission factors in the UK have been reduced around 35\% from 1990 to 2010 (UK Defra \& DECC, 2012). This energy scenario is to explore how the total energy would change if building sector would use electricity instead of gas for heating.

(2) Building type conversion scenario: In urban environment, it may have some reasons to change building types due to economic or social reasons. Then, it may be of interest to investigate change of building types on total energy consumption in cities.

(3) Energy efficient Scenario: In order to reduce carbon emission, it is important to understand how the improvement of energy use efficiency may influence total energy consumption in cities. Thus, this scenario allows analysts studying the influences of change of energy use intensities for different building types. 


\subsection{Optimal simulation of energy consumption based on ABM model in IUEBA}

Implement Agent based model for energy efficiency and human behaviour simulation

The application of this section will facilitate deployment of energy efficient buildings, socioeconomic impacts and energy supply technologies in city design, by developing quantifiable, system-level models that assess their feasibility and implementation in the wider context of socio-economic, and regulatory characteristics of the city. The system-level model will not only enable a more robust scaling-up of the impact of technological innovations up to the city level, but also allow trade-offs between their environment, societal, and economic impacts to inform regulations and policy scenarios.

1. Initiate model environment

In this agent model we apply

- Energy Consumption Source:

lighting, HVAC(heating, ventilation, air-condition, cooling), Equipment,

- Energy Sources:

Gas, (Oil), Electricity, Renewable Energy(Solar, Wind, Biogas, Bio-diesel)

- Environment Sources

Residential, Commercial, Industrial, Transport

In this mobility model we consider people like household employment consumption is the main part of energy consumption of a city People migrate to cities for the following reasons: For better employment, education, treatment, amenities and thus an increased quality of life.

2. Formulation \& Methodology of model simulation

$$
E U I_{\text {msoa }}=\alpha \times e^{\frac{N_{\text {population }}}{c_{p} \times \sum_{j=1}^{M} S_{\text {building }}}}+\beta \times e^{\frac{N_{\text {employment }}}{c_{e} \times \sum_{j=1}^{M} S_{\text {building }}}}
$$

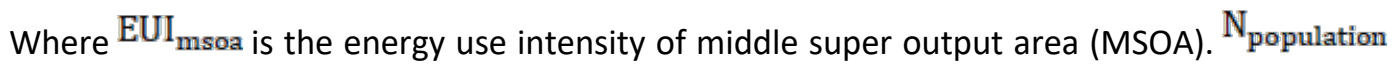
and $\mathrm{N}_{\text {employment }}$ are number of population and employment respectively in MSOA. ${ }^{\text {building }}$ is the floor area of each building type, and ${ }^{M}$ is the number of building types. ${ }_{p}$ and $c_{e}$ adjust the strength of population and Employment that can be determined before regression controlling weights of population and employment.

\section{Results analysis}

\subsection{A case study of London}

London has been used as a case study to explore its building energy patterns with this new integrated IUBEA model environment. In this case-study, London has three spatial scales: LA (local authority), MSOA (middle layer super output area), and LSOA (lower layer super output area). London has 32 local authorities and the city of London is also regarded as a special LA in this study. According to UK census 2001, London has 983 MSOAs and 4765 LSOAs. MSOA and LSOA areas are created based on population and household number. 
Note that there is a change of these MSOA and LSOA areas in 2011 census due to population and household changes.

\begin{tabular}{|c|c|c|}
\hline Theme & Data set & Reference \\
\hline \multirow{3}{*}{ Energy } & Electricity/Gas at borough level & (DECC, 2013c) \\
\hline & Electricity/gas at MSOA and LSOA & (DECC, 2013b) (DECC, 2013a) \\
\hline & $\begin{array}{l}\text { EPC (energy performance certificate) \& DEC } \\
\text { (Display energy certificate) in London }\end{array}$ & (UK DCLG, 2013) \\
\hline \multirow{2}{*}{ Area } & Land use area from UKmap & (UKMap, 2011) \\
\hline & Floor area & (UKMap, 2011) (Choudhary, 2012) \\
\hline \multirow{2}{*}{$\begin{array}{c}\text { Social } \\
\text { economy }\end{array}$} & Population & \multirow[t]{2}{*}{ (London datastore, 2013) } \\
\hline & Household & \\
\hline
\end{tabular}

Table 1. Date source used in this analysis

Table 1 lists the data sets used in this paper. These data can be divided into three categories: energy, area, and social economic factors. The energy data used in this analysis includes regional data and point energy data in London. The regional energy data means the total energy in a specific spatial scale, while the point energy data is the energy data for individual buildings. Both electricity and gas data in domestic buildings in London has good quality from LA to MSOA levels although the energy data in a few number of MSOA areas is unavailable (DECC, 2013a, b, c). For non-domestic energy data, there are full data at LA level from UK DECC. For MSOA gas data in London non-domestic sector, the data quality is also fine except for a small numbers of MSOA areas, whereas the electricity data at MSOA level is almost impossible to use since most of data are unavailable due to disclosure issues (DECC, $2013 b)$. The point energy data in this paper is from UK DCLG, including DEC (display energy certificate) and EPC (energy performance certificate) (UK DCLG, 2013).

\subsection{Results}

As described in section 3, many types of analysis can be implemented. In this section, we only show some results of corresponding the main functions described in section 3 .

\section{Spatial factors analysis}

To show the area percentage of school and offices at MSOA levels in London. Figures 2 present the descriptions of the visualization cases. Cases a and $b$ calculated by two modes absolute/relative only, are used to analyse the scaled /absolute distribution Area of Offices

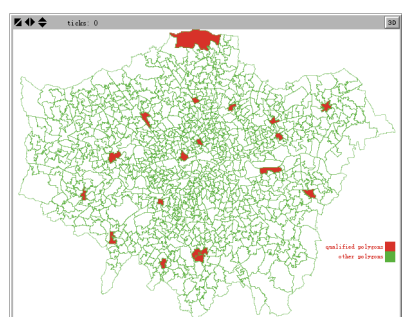

(a)

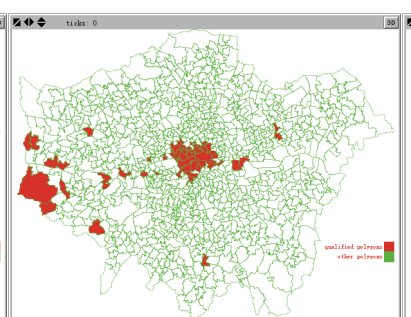

(b)

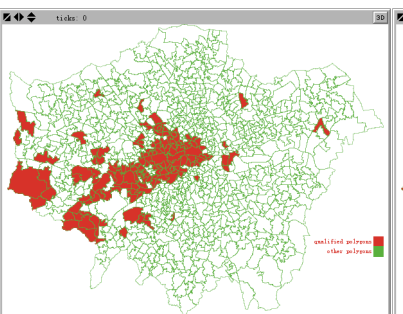

(c)

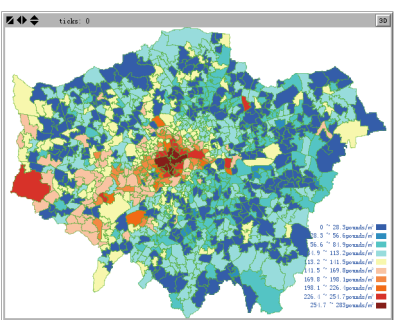

(d)

Figure 3 (a) Retail floor area of MSOA more than 20\% in Greater London. (b) Office floor area of MSOA more than $\mathbf{2 0 \%}$ in Greater London. (c) Office cost of MSOA more than $50 \%$ of 983 polygons in Greater London. (d) Office cost distribution of 983 MLSOA in Greater London

and Schools per MLSOA in London in London. It refers to the operation between properties in polygons, which is very important to quantify the change of some variables per-district. 
Figures 4 Figure 4 (a) (b)the non-domestic electricity is replaced by domestic electricity and the results presents the regression with residential floor area and population. We attempt to explain whether; floor area or employment can explain more on the variations of nondomestic electricity based on $\mathrm{R}^{2}$.
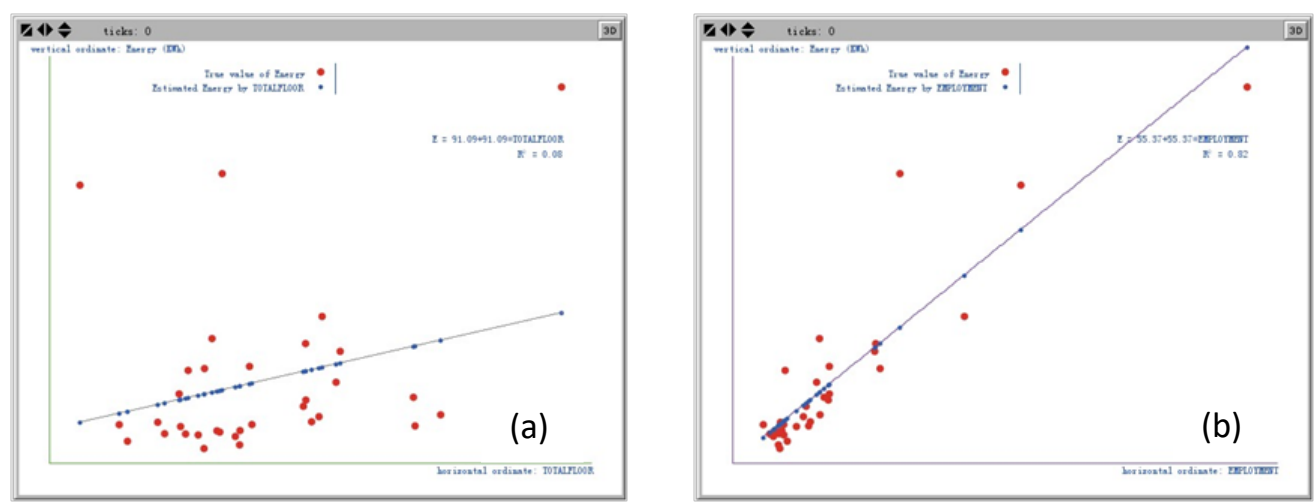

Figure 4 a) regression of domestic-electric/floor-area; b) domestic-electric/population

\section{Spatial distribution analysis}

In the "I-UBEA" combined with GenBUGS software to process the spatial statistical based on Bayes analysis, the model runs 20,000 times, of which "Burn-in Period" is 2000 times. Based on the distribution of the energy density of 13 different types of non-residential buildings in London, this section selects the main proportion of community buildings in London to show the model application. Based on the distribution of the energy density of 13 different types of non-residential buildings in London, this section selects the main proportion of community buildings in London to show the model application. By comparing the density distribution results of 13 building types, the distribution of building energy consumption shows obvious spatial polarization characteristics, and some spatial forms have obvious polarization. Figure 5 shows the distribution of the mean of the posterior distribution of community buildings in the tested 953 MLSOAs. Among them, the high-energy buildings obviously have a concentrated trend, but they do not form a group distribution pattern. Instead, they form a concentrated high-energy-consuming area near the center of the city, and shows distinctly different from the multi-center patterns compare with other types of buildings
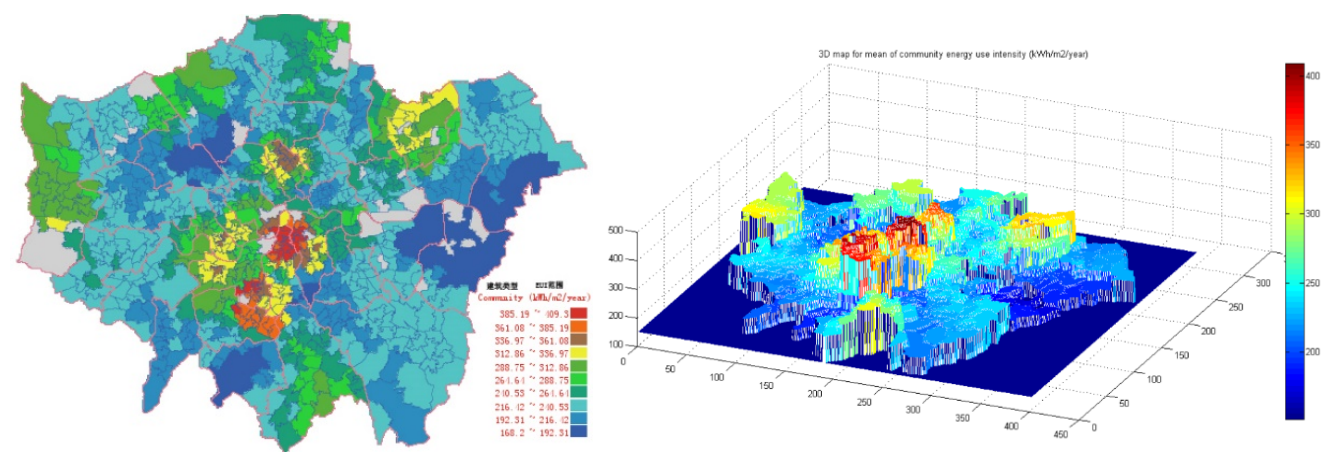

Figure 5 Posterior distributions of calculated area weighted EUls of community building in MLSOAs of London 


\section{Scenarios analysis}

The input data are included all local geography data and historical energy consumption records of recent years. In this section figures 5 presents the comparison results between three scenarios due to electrification in London building sector. The results are based on the distributions of EUI from both DEC and EPC data. Then the results from both base case and electrification scenario distributed in Figure 6.

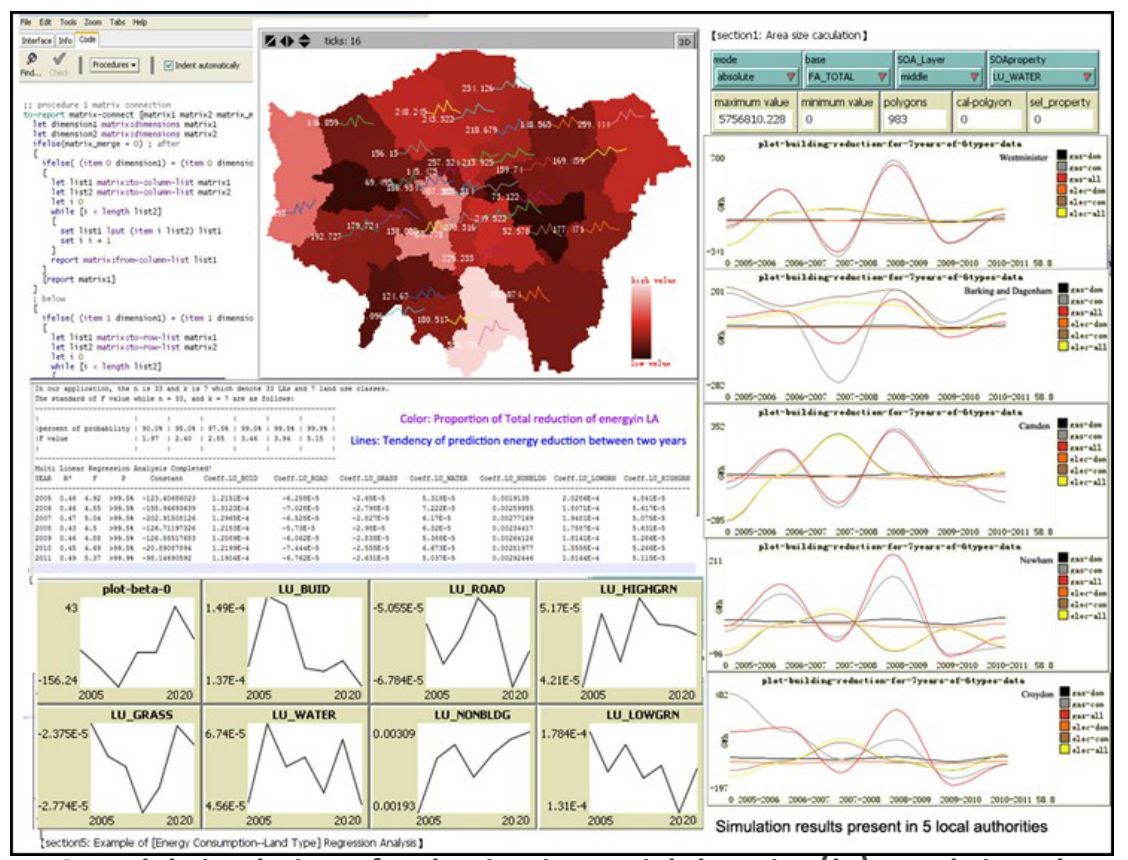

Figure 6 model simulation of reduction in spatial domain (la) and time domain ( 2005-2020)

\section{Agent-based energy models}

Figure 8 shows the people migrates to neighboring MSOA according to accumulation of different energy sources. Weights of each energy source can be set, thus denoting different policies. From the results each MLSOA has its own distribution of resources and population, thus energy use intensity (EUI) in there. Some polygons have higher EUI than others. Statics plots in figure 7 show how the interaction between energy consumptions, sociodemographic characteristics, environmental conditions and human behaviour which all combine in unique ways to create very different energy saving opportunities for each local authority. 


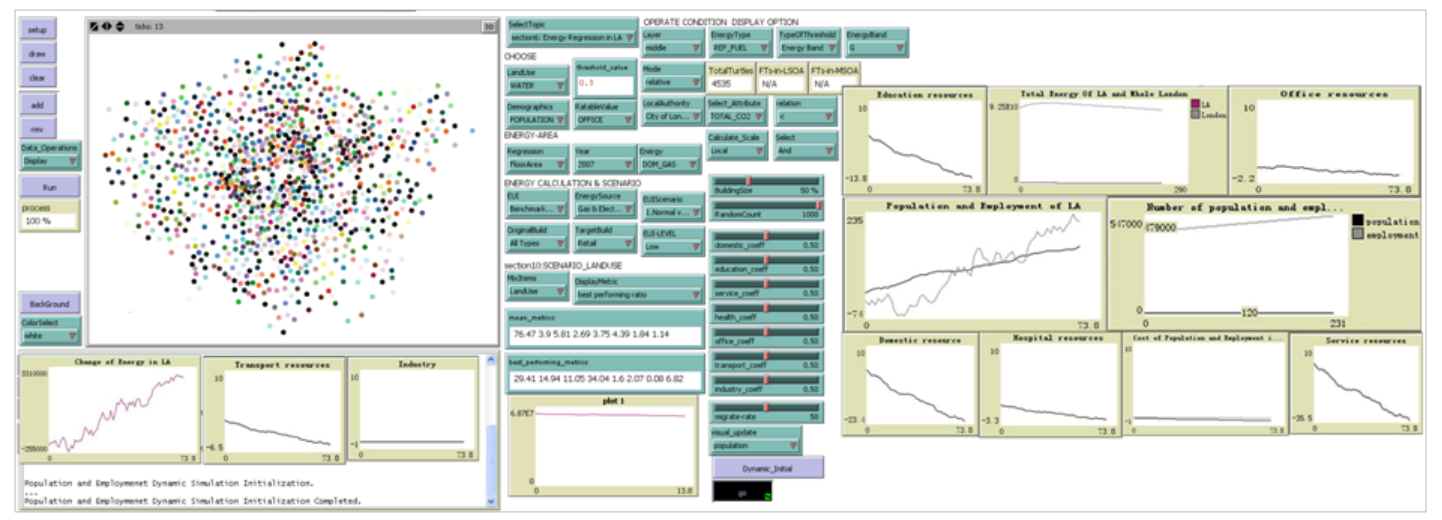

Figure 7 Agent based model for energy prediction in terms of population mobility generated from IUBEA results

\section{Conclusion}

This paper has described a new integrated GIS environment to make thorough analysis of energy performance in city buildings. This energy analysis environment can be used for multiple functions, including spatial data visualization, data query, regression analysis, scenario analysis, energy optimization, and agent-based energy analysis. London has been used as a case study to illustrate its main functions.

The following conclusion also can be drawn from this research:

- Urban scale energy models should consider the effects of spatial dependence when dealing with energy use at urban scale. Or at least, the immediate local environment factors should be analyzed whether there is significant spatial correlation.

- $\quad A B M$ (Agent-based-model) can be a feasible tool to tackle intellectual challenges (uncertainties) in energy modeling. The uncertainty that is caused by occupant behaviors accounts for a significant discrepancy between the predicted and actual energy usage that typically have strong interactions.

- As a result, the research proved the possibility of the integrated approach in simulation, and the analysis of energy-efficiency which is influenced by comprehensive factors including spatial context and human interventions. The final adoption example of London demonstrated that the integrated analysis environment as a feasible tool for building energy consumption have unique advantages and wide applicability.

Funding: This research was funded by National Natural Science Foundation of China, Fund No. 51678178; China Postdoctoral Science Foundation, Fund No. 2018M630361; Postdoctoral Science Foundation of Heilongjiang province, Fund No. AUGA4110003717.

\section{References}

Bose, R. K. (2013). "Energy efficient cities," The World Bank. 2013, 35, 270-283.

Robinson, D. (2011). Computer Modelling for Sustainable Urban Design; Earthscan: London, UK. 
Keirstead, J.; Jennings, A. A review of urban energy system models: Approaches, challenges and opportunities. Renew. Sustain. Energy Rev. 2012, 16, 3847-3866.

Heo Y , Choudhary R , Augenbroe G A.(2012).Calibration of building energy models for retrofit analysis under uncertainty[J]. Energy and Buildings, 47(5):550-560.

Frayssinet, L.; Merlier, L.; Kuznik, F.; Hubert, J. (2018). Modeling the heating and cooling energy demand of urban buildings at city scale. Renew. Sustain. Energy Rev. 81, 2318-2327.

Choudhary, R.; Tian, W. (2014). Influence of district features on energy consumption in nondomestic buildings. Build. Res. Inf. 42, 32-46.

Zhao, F.; Wang, J.; Koritarov, V.(2010).Agent-based modeling of interaction between commercial building stocks and power grid. 2010 IEEE Conference on Innovative Technologies for an Efficient and Reliable Electricity Supply, Waltham, MA, USA, 2729 September; pp. 225-232.

Choudhary, R. (2012). Energy analysis of the non-domestic building stock of Greater London. Building and environment (51), 243-254.

Cheng, V.; Steemers, K. (2011). Modelling domestic energy consumption at district scale: A tool to support national and local energy policies. Environ. Model. Softw. 26, 11861198.

Tian W. (2013). A review of sensitivity analysis methods in building energy analysis[J]. Renewable \& Sustainable Energy Reviews, 20(4):411-419.

UK Defra \& DECC. (2012). Guidelines to Defra/DECC's GHG Conversion Factors for Company Reporting, Produced by AEA for the Department of Energy and Climate Change (DECC) and the Department for Environment, Food and Rural Affairs (Defra), UK; UK Defra \& DECC: London, UK.

DECC.(2013).LLSOA (Lower Layer Super Output Area) Electricity and Gas; DECC (Department of Energy \& Climate Change): London, UK.

EPC.(2013). (Energy Performance Certificate). DEC (Display Energy Certificate) Database Maintained by Landmark, Department for Communities and Local Government, UK; UK DCLG: London, UK. 